\title{
The $c-K I T$ Exon 11 Deletions Cause Resistant to Imatinib Treatment of GISTs through Induction of ULK1-SRC related Autophagy
}

\section{Hao-Chen Wang}

Institute of Clinical Medicine, College of Medicine, National Cheng Kung University

\section{Yu-Tang Chen}

Institute of Clinical Medicine, College of Medicine, National Cheng Kung University

\section{Ya-Chin Hou}

Department of Surgery, College of Medicine, National Cheng Kung University

\section{Chih-Jung Wang}

National Cheng Kung University College of Medicine Department of Surgery

\section{Ying-Jui Chao}

National Cheng Kung University, College of Medicine, Department of Surgery

\section{Yueh-Tsung Lee}

Department of Surgery, Chang-Bing Shaw-Chwan Memorial Hospital

\section{Yan-Shen Shan ( $\nabla$ ysshan@mail.ncku.edu.tw)}

National Cheng Kung University College of Medicine

\section{Research}

Keywords: Gastrointestinal stromal tumor, Autophagy, c-KIT, Imatinib,

Posted Date: April 10th, 2020

DOI: https://doi.org/10.21203/rs.3.rs-22031/v1

License: (9) (i) This work is licensed under a Creative Commons Attribution 4.0 International License. Read Full License 


\section{Abstract}

Background: Exon 11 deletion of $c-K I T$ is the common mutation site with the worst prognosis of gastrointestinal stromal tumors (GISTs). Autophagy has shown to have both protumor and antitumor functions and protect GIST cells from imatinib (IM)-induced apoptosis. In this study, we aimed to explore whether $c-K I T$ exon 11 deletions of GISTs drive refractory to IM treatment through regulation of autophagy.

Methods: 251 GISTs were included in this study to determine the association of $c-K I T$ exon 11 deletions with prognosis and IM resistance. Immunohistochemistry, immunofluorescence, Western blotting were used to assess the correlation between $c-K I T$ exon 11 deletions and autophagy and the underlying mechanism. We also studied the antitumor effect of IM in combination with the autophagy inhibitor chloroquine (CQ) in GIST62 cell derived xenograft mice model.

Results: GISTs with c-KIT exon 11 deletions had worse survival and IM-resistant phenotype and exhibited higher autophagy levels as compared with GISTs harboring wild type or other $c-K I T$ mutations. In addition, LC3-II expression strongly increased in GIST cell lines carrying c-KIT exon 11 deletions. Treated with autophagy inhibitors or knockdown of the autophagy-related gene ATG5 effectively inhibit GIST cells viability. Co-treatment with IM and CQ significantly suppressed tumor growth as compared with either agent alone, suggesting that autophagy protect GIST cells from IM-induced cell death both in vitro and in vivo. Furthermore, c-KIT exon 11 deletions can induce autophagy by activating SRC and ULK1. SRC inhibitor can block this action.

Conclusion: Our study clearly demonstrates that KIT exon 11 deletions induce ULK1-SRC related autophagy is one of reasons for imatinib resistance clinically. Combination with autophagy inhibitor shows significant promise in treatment for imatinib refractory GISTs.

\section{Background}

Gastrointestinal stromal tumor (GIST), originating from the lineage of interstitial cells of Cajal (ICC) [1], is the most common mesenchymal neoplasm of the gastrointestinal tract with the annual incidence rate of approximately 10-15 cases per million population [2]. Currently, surgery is the mainstay of treatment for patients with resectable GIST; however, approximately $50 \%$ of GIST patients develop recurrence or metastasis after complete resection of the primary tumor [3]. Imatinib (IM), a selective tyrosine kinase inhibitor is the standard of care for patients with unresectable or metastatic GISTs. It has led to significant improvements in survival of GIST patients. However, the majority of patients inevitably acquire drug resistance, highlighting the urgent need for identification and targeting of the mechanisms underlying resistance to IM. 
GISTs are typically immunoreactive for KIT, also known as CD117. This protein is observed in over $95 \%$ of GISTs, and is the most specific and sensitive marker in differentiating GISTs from other mesenchymal tumors in the GI tract [4]. KIT is a $145 \mathrm{kD}$ transmembrane glycoprotein and serves as a receptor for stem cell factor (SCF). Binding of SCF activates KIT downstream pathways such as MAPK, AKT, SRC, and STAT3, consequently regulating a variety of cell functions, including proliferation, survival, apoptosis, and motility $[5,6]$. About $75-80 \%$ of sporadic GISTs have $c-K I T$ mutations, which have been considered a major driving force behind tumor development [7]. The most common mutation in KIT affects the juxtamembrane region encoded by exon 11 . This region, just inside the cell membrane, is a helical domain that functions to inhibit KIT kinase activity in the absence of ligands [8]. Mutagenesis studies on $c-K I T$ have demonstrated that mutations of this domain disrupt this function, allowing ligandindependent receptor dimerization and constitutional activation. Several reports have shown that $c-K I T$ exon 11 deletions confer worse prognosis and predispose GISTs to metastasis [9]. Codons 557 and 558 are the most common sites for deletion in $c-K I T$ exon 11 and $c-K I T$ exon 11 deletions involving codons 557-558 are highly associated with liver metastasis of GISTs [10].

Autophagy is an evolutionarily conserved pathway of cellular catabolic degradation in response to starvation or stress. It can degrade and recycle long-lived proteins and organelles, serving as protein quality controller [11]. Based on its functions to keep cellular homeostasis, autophagy can act as a crucial adaption pathway that can promote tumorigenesis and survival of cancer cells under nutrient defeat. Accumulating evidence has indicated that autophagy has protumor functions. For examples, autophagy can enhance the tolerance of stress, which maintains tumor cell survival [12]. Cancer cells have high metabolic demands and exposure to metabolic stress is shown to impair survival in autophagy-deficient cells in vitro. Hypoxia and nutrient deprivation can activate the autophagy for recycling of ATP and to maintain cellular biosynthesis and survival [13]. In genetically engineered mouse models driven by Kras or Braf mutation, depletion of ATG5 and ATG7 resulted in diminished tumor burden [14]. In addition, autophagy can also maintain activity of pancreatic cancer stem cells that contribute to chemoresistance of cancer cells, and blockade of autophagy potentiates antitumor effect of gemcitabine [15], suggesting that autophagy can function as a tumor promoter. Previous research has shown that inhibition of autophagy increases drug sensitivity in GIST cells with c-KIT exon 11 deletions [16], raising that autophagy acts as a survival mechanism. Therefore, in this study, we were interested in determining whether $c-K I T$ exon 11 deletions cause resistant to imatinib treatment in GISTs by up-regulating autophagy.

\section{Methods}

\section{Cell lines}

The human GIST cell lines, GIST62 with KIT-negative and GIST48 with a homozygous c-KIT exon 11 V560D mutation and a heterozygous c-KIT exon 17 D820A mutation, were gifts from Dr. Li-Tzong Chen (National Institute of Cancer Research, National Health Research Institutes, Tainan, Taiwan). The human GIST cell line with exon 11 deletion, GIST-T1 was purchased from Cosmo Bio Co., Ltd. Both GIST62 and 
GIST48 were maintained in RPMI 1640 medium (Hyclone) supplemented with 15\% fetal bovine serum (FBS; Thermo Fisher Scientific), 15 mM HEPES (Biological Industries), 2 mM L-glutamine (Caisson Laboratories), $1 x$ antibiotic-antimycotic solution (1000 units/L penicillin, $2.5 \mu \mathrm{g} / \mathrm{L}$ amphotericin $\mathrm{B}$, and $1000 \mu \mathrm{g} / \mathrm{L}$ streptomycin; Caisson Laboratories). GIST-T1 cells were maintained in Dulbecco's Modified Eagles Medium (Hyclone) supplemented with 10\% FBS (Thermo Fisher Scientific), 15 mM HEPES (Biological Industries), $2 \mathrm{mM}$ L-glutamine (Caisson Laboratories), $1 \times$ antibiotic-antimycotic solution (1000 units/L penicillin, $2.5 \mu \mathrm{g} / \mathrm{L}$ amphotericin B, and $1000 \mu \mathrm{g} / \mathrm{L}$ streptomycin; Caisson Laboratories). All cell lines cultured in a humidified atmosphere with $5 \%$ of $\mathrm{CO}_{2}$ at $37^{\circ} \mathrm{C}$.

\section{Transfection}

GIST62 cells in 6-well plates were transfected with $2 \mu \mathrm{g}$ of plasmids, including pcDNA3.1b-KIT exon 11 $\triangle 557-558$, pcDNA3.1b-KIT wild type, pcDNA3.1b-KIT exon11 V560D, and empty pcDNA3.1b vector as control using lipofectamine 2000 (Invitrogen) according to the manufacturer's protocol. After transfection for 48 hours, we harvested cells for further study. We also used geneticin $(800 \mu \mathrm{g} / \mathrm{mL})$ for selection to establish stable cell lines.

\section{Protein Extraction}

Cells in culture dishes were washed with ice-cold PBS and were collected to microcentrifuge tube using cell scraper. The cells were lysed with ice-cold RIPA cell lysis buffer (Millipore Corporation) containing protease inhibitor cocktail (Millipore Corporation) and phosphatase inhibitor cocktail (Sigma-Aldrich). The cell lysate was centrifuged at $13,000 \mathrm{rpm}$ at $4{ }^{\circ} \mathrm{C}$ for 30 minutes, and then the supernatant was collected to a new $1.5 \mathrm{~mL}$ microcentrifuge tube. The protein concentration of the samples was measured by BCA protein assay (Thermo Fisher Scientific), and all samples were stored at $-80^{\circ} \mathrm{C}$.

\section{Western Blotting}

Equal amounts of protein in $1 \times$ SDS loading buffer were heated at $95^{\circ} \mathrm{C}$ for 10 minutes. Samples were separated by 8 or $10 \%$ SDS-PAGE and transferred to Immobilon-P membranes (Millipore Corporation) at $100 \mathrm{~V}$ for 100 minutes. The membrane was then blocked with $5 \%$ milk diluted in $0.05 \%$ TBST for 1 hour at room temperature. After blocking, membrane was probed by primary antibodies diluted in $0.05 \%$ TBST overnight at $4{ }^{\circ} \mathrm{C}$. Next day, the membrane was washed 10 minutes for three times with $0.05 \%$ TBST followed by incubation with horseradish peroxidase (HRP)-conjugated secondary antibodies for 1 hour at room temperature. The membrane was washed 10 minutes for three times with $0.05 \%$ TBST. The blots signal was developed by using Immobilon Western Chemiluminescent HRP Substrate (Millipore Corporation) according to the manufacturer's instruction and captured by Biospectrum Imaging System (UVP). 


\section{Immunohistochemical (ihc) Analysis}

The formalin-fixed paraffin embedded tissues were cut into $5 \mu$ m-thick sections. Antigen retrieval was performed by autoclaving the sections at $121^{\circ} \mathrm{C}$ for 10 minutes in sodium citrate buffer ( $\left.10 \mathrm{mM}, \mathrm{pH} 6.0\right)$. After blocking with the blocking buffer (Thermo Fisher Scientific) at room temperature for 30 minutes, the sections were incubated with specific primary antibodies at $4{ }^{\circ} \mathrm{C}$ overnight and then with biotinylated secondary antibodies at room temperature for 30 minutes. The immunoreaction was visualized using a DAB chromogen system (DAKO). The cell nuclei were stained with hematoxylin.

\section{Immunofluorescence (if) Staining}

Cells $\left(3 \times 10^{4}\right.$ cells/well) were seeded in an 8-well chamber slide (Millipore Corporation). After 24 hours of growth, cells were fixed in $4 \%$ paraformaldehyde for 10 minutes and permeabilized in $0.05 \%$ Triton X-100 for 15 minutes. Cells were then blocked in Super Blocking Buffer (Pierce) for 1 hour and incubated with primary antibodies overnight. Next day, cells were incubated with fluorochrome-conjugated secondary antibodies for 1 hour at room temperature. Nuclei were counter-stained with DAPI. All the images were visualized using a fluorescence microscope (BX51, OLYMPUS).

\section{Animal Model}

Seven weeks old male NOD/SCID mice were subcutaneously injected with GIST62 V cells or GIST62 $\Delta$ cells $\left(4 \times 10^{6}\right.$ cells in $50 \mu \mathrm{L}$ serum free medium with $50 \mu \mathrm{L}$ matrix gel). The tumor volume was calculated using the formula of (length $\times$ width $\left.{ }^{2}\right) / 2$. When tumor volume was $>100 \mathrm{~mm}^{3}$, the drug treatments started (every 3 days for 15 days). After the treatments, we sacrificed these mice and collected the tumors. During this study, we raised and cared the animals according to the guidelines set up by the National Science Council, Taiwan. The Institutional Animal Care and Use Committee in NCKU Laboratory Animal Center approved animal experiments.

\section{Cell Viability Analysis}

GIST62V and GIST62 $\Delta$ cells $\left(8 \times 10^{3}\right.$ cells/well) were seeded on a 96 -well plate. After drug treatment, medium was removed and the cells were fixed with methanol for 10 minutes and stained with $0.05 \%$ of methylene blue for 1 hour. The cells were then washed with water and air-dried overnight. Hydrochloric acid $(0.5 \mathrm{~N})$ was added to each well to dissolve the methylene blue stain and the absorbance was measured with the SpectraMax M5 microplate reader (Molecular Device) at $595 \mathrm{~nm}$ wavelength and normalized to the DMSO control group.

\section{Patients And Specimens}


Two hundred and fifty-one GISTs from National Cheng Kung University Hospital (NCKUH) were included, and their clinical records were retrospectively analyzed in this study. The study protocol was reviewed and approved by the Institutional Review Board of NCKUH (ER-104-157). Anonymous archived samples of GISTs were obtained from Human Biobank of NCKUH.

\section{Statistical analysis}

Statistical evaluation was conducted using GraphPad Prism software version 5.01 (GraphPad, Inc.). Values are expressed as means \pm SEM. Differences between groups were determined by Student's $t$ test and ANOVA. The survival probability was calculated with the Kaplan-Meier method. $P$ value less than 0.05 was considered to be statistically significant $\left({ }^{\star} p<0.05,{ }^{\star \star} p<0.01,{ }^{\star \star *} p<0.001\right)$.

\section{Results}

\section{$c-K I T$ exon 11 deletions correlate with poor prognosis and IM resistance}

Previous studies have reported that GISTs harboring mutations in c-KIT exon 11, especially deletions, exhibit more aggressive behavior and enhanced propensity for liver metastasis $[9,10,17]$. Consistently, our clinical data show that, when the GIST patients were divided into four groups: wild type $c-K I T, c-K I T$ exon 11 deletions, other c-KIT exon 11 mutations, and other c-KIT mutations, those with c-KIT exon 11 deletions had more metastasis rate, recurrence rate (Table 1). Furthermore, those patients also had the worst disease-free survival (Fig. 1A). We further compared the responses to IM of the four groups. GIST patients with c-KIT exon 11 deletions had the lower response rate than those in the other three groups (Fig. 1B). These results indicate that $c-K I T$ exon 11 deletions are associated with poor prognosis in GISTs.

\section{GISTs with c-KIT exon 11 deletions exhibit high levels of autophagy}

Autophagy has been widely implicated in mechanisms governing tumor development and chemoresistance [18]. In GIST, autophagy can protect tumor cells from IM-induced apoptosis [16]. To elucidate whether autophagy is involved in $c-K I T$ exon 11 deletions-induced malignant properties and drug resistance of GIST cells, the correlation between $c-K I T$ exon 11 deletions and autophagy was evaluated. We compared the autophagosomal marker LC3-II expression in human GIST specimens with wild-type $c-K I T, c-K I T$ exon 11 deletion, and $c-K I T$ exon 11 V560D mutation. We found GISTs harboring $c$ KIT exon 11 deletion had the highest expression of LC3-II (Fig. 2A). IHC staining for LC3-II also showed that GISTs harboring $c-K I T$ exon 11 deletion expressed a higher level of LC3 puncta than that with wildtype $c-K I T$ (Fig. 2B). Quantification of LC3 puncta levels in GIST specimens revealed a positive correlation between $c-K I T$ exon 11 deletion and autophagy (Fig. 2C). To further confirm the positive correlation 
between autophagy and $c-K / T$ exon 11 deletions, we established stable transfected cell lines from KITnegative GIST62 cells: vector control GIST62V cells and GIST62D cells that carry c-KIT exon 11 deletion. We observed that LC3-II expression was higher in GIST62D cells and GIST-T1 cells but lower in GIST62V cells and GIST48 cells (Fig. 2D). IF staining for LC3 showed a higher level LC3 puncta in GIST62D cells than in GIST62V cells (Fig. 2E). Taking together, we prove that $c-K I T$ exon 11 deletions upregulate autophagy in GIST cells.

\section{Autophagy mediates $c$-KIT exon 11 deletions-induced GIST cell growth}

We next determined the effect of autophagy upregulation in GIST cells with $c-K I T$ exon 11 deletions. High expression of LC3-II and reduced expression of the autophagy marker SQSTM1/p62 indicating autophagy activation [19] was accompanied by increased expression of the cell proliferation marker Ki-67 in GIST62D cells but not in GIST62V cells (Fig. 3A), while there was no difference in apoptosis between GIST62D cells and GIST62V cells (supplemental Fig. 1). As expected, GIST62D cells also showed greater cell growth potential and mitotic activity than GIST62V cells did (Fig. 3B, 3C, and supplemental Fig 2). When autophagy was blocked by various pharmacological inhibitors including Bafilomycin A1 (Baf A1), chloroquine (CQ), and 3-methyladenine (3-MA), the viability of GIST62D cells was decreased in a dosedependent fashion (Fig. 3D-F). A similar result also obtained after the core autophagy gene ATG5 inactivated by RNA interference in GIST62D cells (Fig. 3G). These data indicate that autophagy is required for $c$-KIT exon 11 deletions-mediated cell growth in GISTs.

\section{Inhibition of autophagy reduces tumor growth and enhances IM cytotoxicity}

To investigate the in vivo relevance of the in vitro observations, we used a xenograft model derived from GIST62 cells. We first compared the tumor growth rate between GIST62V cells and GIST62D cells by subqutaneous injection of the cells into NOD/SCID mice. The growth rate of GIST62 $\Delta$ tumors was significantly higher than that of GIST62V tumors (Fig. 4A). Six weeks after injection, mice were sacrified and tumors were collected. The size and the weight were greater for GIST62 $\Delta$ tumors as compared with GIST62V tumors (Fig. 4B and 4C), indicating that $c-K I T$ exon 11 deletion promotes GIST tumor growth. Western blotting using collected tumors confirmed that LC3-II was highly expressed in GIST62 $\Delta$ tumors but not in GIST62V tumors (Fig. 4D). We next assessed the effect of autophagy blockade on tumor growth and the therapeutic efficacy of IM. GIST62D cells were subqutaneously inoculated into NOD/SCID mice to form tumors. When the volume reached around $100 \mathrm{~mm}^{3}$, mice were randomized to receive a 2week course of twice-weekly treatment with vehicle, CQ, IM, or in combination. Two weeks after cell inoculation, the mean volume of tumors treated with vehicle was about $450 \mathrm{~mm}^{3}$. Compared to CQ or IM alone treatment that decreased the mean tumor volume to about $300 \mathrm{~mm}^{3}$, CQ combined with IM 
treatment could more effectively reduce the tumor volume to about $120 \mathrm{~mm}^{3}$ (Fig. 4E). Similar results were also seen in tumor size and tumor weight measured at the end point of the experiment (Fig. 4F and $4 \mathrm{G})$. These in vivo results indicate that inhibiting autophagy can not only retard tumor growth but also enhance the susceptibility of GIST cells to IM.

\section{ULK1 and SRC are involved in c-KIT exon 11 deletions- induced autophagy}

To decipher the mechanism by which c-KIT exon 11 deletions induce autophagy in GIST cells, we first identified the autophagy-related proteins whose expression altered. We found that $c-K I T$ exon 11 deletion mutation did not affect the expression of ATG5, ATG7, and Beclin 1 (Fig. 5A). However, ULK1 Serine-556 phosphorylation that contributes to autophagy activation increased, while ULK1 Serine-758 phosphorylation that deactivates ULK1 decreased in GIST62D cells as compared with GIST62V cells (Fig. $5 \mathrm{~A}$ ). The nonreceptor tyrosine kinase SRC is one of the major signaling molecule located downstream of KIT and has been reported to be involved in autophagy activation [20-22]. A previous study revealed that phosphorylation of mATG9 at Tyr8 by SRC functionally cooperates with Ser14 phosphorylation by ULK1 to regulate MATG9 trafficking and redistribution for autophagy initiation [23]. We therefore observed the activation of SCR in GIST cells with different $c-K I T$ mutations by analyzing its phosphorylation and evaluated the correlation between SRC phosphorylation and autophagy. Enhanced expression of phosphorylated SRC (pSRC) and LC3-II observed in GIST62D cells and GIST T1 cells (Fig. 5B). GIST62D cells expressed the highest levels of pSCR and LC3-II than the other GIST62 transfectants, including GIST62V cells, GIST62 WT cells that overexpress wile-type KIT, and GIST62 V560D cells that carry c-KIT exon 11 V560D mutation (Fig. 5C). Treatment with a specific SRC kinase inhibitor bosutinib in GIST62D cells caused decreased SRC phosphorylation with a concomitant suppression in LC3-II expression. These data suggest that $c-K I T$ exon 11 deletions upregulate autophagy by activating ULK1 and SRC.

\section{Discussion}

Autophagy is not only a critical catabolic process essential for cellular homeostasis but has also been linked to cancer, playing a dual role in tumor cell survival and death [24]. In GISTs, c-KIT exon 11 deletion associates with worse prognosis and confers a more aggressive phenotype $[9,10]$. However, the correlation between $c-K I T$ exon 11 deletion and autophagy in GIST progression is not clear. In this study, we observed that GISTs with $c-K I T$ exon 11 deletion mutations had a higher rate of resistance to IM than that with wild type $c-K I T$ or other $c-K I T$ mutations. The presence of $c-K I T$ exon 11 deletion mutations showed a significant positive correlation with autophagy levels in GIST clinical specimens and cell lines. Blockade of autophagy effectively suppressed GIST growth and enhanced the antitumor activity of IM in vitro and in vivo, suggesting the requirement of autophagy for $c-K I T$ exon 11 deletion mutations-induced aggressiveness in GISTs. 
Our results show that SRC was highly phosphorylated in GIST cells with $c-K I T$ exon 11 deletion but not in GIST cells with $c-K I T$ exon 11 V560D or wild type $c-K I T$. The phosphorylation of SRC might be independent of KIT autophosphorylation but affected by different mutations-induced receptor conformational changes that can selectively recruit different intracellular signaling pathways $[25,26]$. Furthermore, some studies have found that autophagy has an important role in SRC-mediated oncogenic activities of cancer cells. For example, autophagy promotes focal adhesion disassembly and cell motility of metastatic breast cancer cells through the paxillin-LC3 interaction which is regulated by SRC [22]. Activation of the SRC/STAT3 pathway can induce heme oxygenase- 1 expression by promoting autophagy to protect breast cancer cells from doxorubicin-induced cytotoxicity [27]. Consistently, in this study, we found that SRC phosphorylation positively correlated with autophagy induction, and inhibition of SRC could reduce autophagy levels, suggesting that SRC can act as a positive regulator of autophagy induced by $c-K I T$ exon 11 deletion mutations in GIST cells.

Many tyrosine kinase inhibitors have been developed to target KIT activity to treat GIST, such as IM, sunitinib, regorafenib, sorafenib, nilotinib, ponatinib, masitinib, dasatinib, and dovitinib [28]. IM is the first line drug with significant improvement of GIST patients' survival, but some GIST patients with kit exon 11 deletion mutations show refractory to IM treatment soon. These patients should receive second line drug treatment by sunitinib. Our study proved combination of IM and autophagy inhibitor can inhibit tumor growth. Furthermore, SRC inhibitor can reduce the autophagy activity induced by kit exon 11 deletion, which meant combination with IM and SRC inhibitor might be used for those patients refractory to IM treatment. Gupta A et al also had proposed similar strategy to enhance GIST cytotoxicity and to diminish both cellular quiescence and acquired resistance in GIST patients [16]. Recently, the Hsp90AA1 inhibitor 17-AAG is another drug to inhibit KIT pathway, which blocks heat shock protein and thus promotes autophagy to degrade KIT protein [29]. Since 17-AAG exert a different mechanism to inhibit KIT, whether the combination of autophagy inhibitors with this drug has synergistic effect in GIST treatment need to be studied.

Many studies show that knockdown of autophagy-associated genes can inhibit cancer cells growth or increase anticancer efficiency. In our results, we found that inhibition of autophagy could inhibit the growth of GIST62 $\Delta$ cells. Inhibition of autophagy may be a potential strategy to treat GISTs. Additionally, cotreament with autophagy inhibitors becomes a more common option in cancer treatment. Effects of the combination of chemotherapy and the autophagy inhibitor CQ in many cancers have been assessed in pre-clinical studies $[14,30,31]$. These studies prove that inhibition of autophagy can serve as reliable method to treat cancers.

\section{Conclusion}

IM resistance remains an important issue in GIST treatment. Our study showed that $c-K I T$ exon 11 deletions enable GIST cells to obtain IM resistance through autophagy induction. Activation of ULK1 and $\mathrm{SRC}$ is required for $c-K I T$ exon11 deletions-induced autophagy. Inhibition of autophagy can improve IM 
treatment in GISTs with $c-K I T$ exon11 deletions. Therefore, we provide a new strategy for treatment of GISTs patients with $c-K I T$ exon 11 deletions to improve the prognosis.

\section{Abbreviations}

Baf A1: Bafilomycin A1

CQ: chloroquine

Gl: gastrointestinal

GIST: gastrointestinal stromal tumor

ICC: interstitial cells of Cajal

IF: immunofluorescence

IHC: immunohistochemical

IM: imatinib

NCKUH: National Cheng Kung University Hospital

SCF: stem cell factor

3-MA: 3-methyladenine

\section{Declarations}

\section{Acknowledgements}

We sincerely thank Dr. Li-Tzong Chen (National Institute of Cancer Research, National Health Research Institutes, Tainan, Taiwan) for providing the human GIST cell lines GIST62 and GIST48, and the Immunobiology Core Laboratory of NCKUH for the IF imaging analysis.

\section{Funding}

This work was supported by the National Cheng Kung University-Show Chwan Health Care System R\&D Project (NCKUSCMH10601 and NCKUSCMH10703).

\section{Availability of data and materials}

The datasets supporting the conclusions of this article are included within the article.

\section{Authors' contributions}


HCW, YTL, and YSS designed and conceived the study. HCW, YTC, YCH, CJW and YJC performed the experiments and analyzed the data. HCW and YTC wrote the manuscript. YTL and YSS reviewed and revised the manuscript. All authors read and approved the final manuscript.

\section{Ethics approval and consent to participate}

All procedures performed in this study involving human tissues and data were approved by the Institutional Review Board of NCKUH (IRB number: ER-104-157). The animal care and experimental procedures were conducted in accordance with the regulations of the Institutional Animal Care and Use Committee of NCKU.

\section{Consent for publication}

All authors have reviewed the manuscript and given consent for publication.

\section{Competing interests}

All authors declare that they have no competing interests.

\section{References}

1. Hirota S, Isozaki K, Moriyama Y, Hashimoto K, Nishida T, Ishiguro S, Kawano K, Hanada M, Kurata A, Takeda $\mathrm{M}$ et al: Gain-of-function mutations of c-kit in human gastrointestinal stromal tumors. Science (New York, NY) 1998, 279(5350):577-580.

2. Ho MY, Blanke CD: Gastrointestinal stromal tumors: disease and treatment update. Gastroenterology 2011, 140(5):1372-1376 e1372.

3. Gold JS, DeMatteo RP: Combined surgical and molecular therapy: the gastrointestinal stromal tumor model. Annals of surgery 2006, 244(2):176.

4. Zhao X, Yue C: Gastrointestinal stromal tumor. Journal of gastrointestinal oncology 2012, 3(3):189.

5. Corless CL, Fletcher JA, Heinrich MC: Biology of gastrointestinal stromal tumors. Journal of Clinical Oncology 2004, 22(18):3813-3825.

6. Miettinen M, Lasota J: Gastrointestinal stromal tumors (GISTs): definition, occurrence, pathology, differential diagnosis and molecular genetics. Pol J Pathol 2003, 54(1):3-24.

7. Hirota S, Isozaki K, Moriyama Y, Hashimoto K, Nishida T, Ishiguro S, Kawano K, Hanada M, Kurata A, Takeda M: Gain-of-function mutations of c-kit in human gastrointestinal stromal tumors. Science 1998, 279(5350):577-580.

8. Kitamura Y, Hirotab S: Kit as a human oncogenic tyrosine kinase. Cellular and molecular life sciences: CMLS 2004, 61(23):2924-2931.

9. Andersson J, Bumming P, Meis-Kindblom JM, Sihto H, Nupponen N, Joensuu H, Oden A, Gustavsson B, Kindblom LG, Nilsson B: Gastrointestinal stromal tumors with KIT exon 11 deletions are associated with poor prognosis. Gastroenterology 2006, 130(6):1573-1581. 
10. Wang HC, Li TY, Chao YJ, Hou YC, Hsueh YS, Hsu KH, Shan YS: KIT Exon 11 Codons 557-558 Deletion Mutation Promotes Liver Metastasis Through the CXCL12/CXCR4 Axis in Gastrointestinal Stromal Tumors. Clinical cancer research : an official journal of the American Association for Cancer Research 2016, 22(14):3477-3487.

11. Kenific CM, Debnath J: Cellular and metabolic functions for autophagy in cancer cells. Trends in cell biology 2015, 25(1):37-45.

12. Degenhardt K, Mathew R, Beaudoin B, Bray K, Anderson D, Chen G, Mukherjee C, Shi Y, Gelinas C, Fan $Y$ et al: Autophagy promotes tumor cell survival and restricts necrosis, inflammation, and tumorigenesis. Cancer Cell 2006, 10(1):51-64.

13. Semenza GL: HIF-1: upstream and downstream of cancer metabolism. Current opinion in genetics \& development 2010, 20(1):51-56.

14. Lu Z, Luo RZ, Lu Y, Zhang X, Yu Q, Khare S, Kondo S, Kondo Y, Yu Y, Mills GB et al: The tumor suppressor gene ARHI regulates autophagy and tumor dormancy in human ovarian cancer cells. The Journal of clinical investigation 2008, 118(12):3917-3929.

15. Yang MC, Wang HC, Hou YC, Tung HL, Chiu TJ, Shan YS: Blockade of autophagy reduces pancreatic cancer stem cell activity and potentiates the tumoricidal effect of gemcitabine. Molecular cancer 2015, 14:179.

16. Gupta A, Roy S, Lazar AJ, Wang WL, McAuliffe JC, Reynoso D, McMahon J, Taguchi T, Floris G, Debiec-Rychter $\mathrm{M}$ et al: Autophagy inhibition and antimalarials promote cell death in gastrointestinal stromal tumor (GIST). Proceedings of the National Academy of Sciences of the United States of America 2010, 107(32):14333-14338.

17. Lasota J, Jasinski M, Sarlomo-Rikala M, Miettinen M: Mutations in exon 11 of c-Kit occur preferentially in malignant versus benign gastrointestinal stromal tumors and do not occur in leiomyomas or leiomyosarcomas. The American journal of pathology 1999, 154(1):53-60.

18. Amaravadi R, Kimmelman AC, White $\mathrm{E}$ : Recent insights into the function of autophagy in cancer. Genes \& development 2016, 30(17):1913-1930.

19. Klionsky DJ, Abdelmohsen K, Abe A, Abedin MJ, Abeliovich H, Acevedo Arozena A, Adachi H, Adams $\mathrm{CM}$, Adams PD, Adeli $\mathrm{K}$ et al: Guidelines for the use and interpretation of assays for monitoring autophagy (3rd edition). Autophagy 2016, 12(1):1-222.

20. Chen SY, Chiu LY, Maa MC, Wang JS, Chien CL, Lin WW: zVAD-induced autophagic cell death requires c-Src-dependent ERK and JNK activation and reactive oxygen species generation. Autophagy 2011, 7(2):217-228.

21. Moon SY, Kim HS, Nho KW, Jang YJ, Lee SK: Endoplasmic reticulum stress induces epithelialmesenchymal transition through autophagy via activation of c-Src kinase. Nephron Exp Nephrol 2014, 126(3):127-140.

22. Sharifi MN, Mowers EE, Drake LE, Collier C, Chen H, Zamora M, Mui S, Macleod KF: Autophagy Promotes Focal Adhesion Disassembly and Cell Motility of Metastatic Tumor Cells through the Direct Interaction of Paxillin with LC3. Cell Rep 2016, 15(8):1660-1672. 
23. Zhou C, Ma K, Gao R, Mu C, Chen L, Liu Q, Luo Q, Feng D, Zhu Y, Chen Q: Regulation of mATG9 trafficking by Src- and ULK1-mediated phosphorylation in basal and starvation-induced autophagy. Cell Res 2017, 27(2):184-201.

24. Kaur J, Debnath J: Autophagy at the crossroads of catabolism and anabolism. Nat Rev Mol Cell Biol 2015, 16(8):461-472.

25. Casteran N, De Sepulveda P, Beslu N, Aoubala M, Letard S, Lecocq E, Rottapel R, Dubreuil P: Signal transduction by several KIT juxtamembrane domain mutations. Oncogene 2003, 22(30):4710-4722.

26. Barone I, Brusco L, Fuqua SA: Estrogen receptor mutations and changes in downstream gene expression and signaling. Clin Cancer Res 2010, 16(10):2702-2708.

27. Tan Q, Wang H, Hu Y, Hu M, Li X, Aodengqimuge, Ma Y, Wei C, Song L: Src/STAT3-dependent heme oxygenase-1 induction mediates chemoresistance of breast cancer cells to doxorubicin by promoting autophagy. Cancer Sci 2015, 106(8):1023-1032.

28. Serrano C, Marino-Enriquez A, Tao DL, Ketzer J, Eilers G, Zhu M, Yu C, Mannan AM, Rubin BP, Demetri GD et al: Complementary activity of tyrosine kinase inhibitors against secondary kit mutations in imatinib-resistant gastrointestinal stromal tumours. Br J Cancer 2019, 120(6):612-620.

29. Hsueh YS, Yen CC, Shih NY, Chiang NJ, Li CF, Chen LT: Autophagy is involved in endogenous and NVP-AUY922-induced KIT degradation in gastrointestinal stromal tumors. Autophagy 2013, 9(2):220233.

30. Liang XH, Jackson S, Seaman M, Brown K, Kempkes B, Hibshoosh H, Levine B: Induction of autophagy and inhibition of tumorigenesis by beclin 1. Nature 1999, 402(6762):672-676.

31. Mathew R, Karp CM, Beaudoin B, Vuong N, Chen G, Chen HY, Bray K, Reddy A, Bhanot G, Gelinas C et al: Autophagy suppresses tumorigenesis through elimination of p62. Cell 2009, 137(6):1062-1075.

\section{Table 1}

Table 1. Characteristics and tumor mutation profiles of 251 GIST patients 
TABLE 5. Tumors characteristics according to KIT and PDGFRA mutations ( $\mathrm{n}=\mathbf{2 5 1}$ )

\begin{tabular}{|c|c|c|c|c|c|}
\hline Variable & $\begin{array}{c}\text { No mutation } \\
(\mathrm{n}=72)\end{array}$ & $\begin{array}{l}\text { Exon } 11 \text { deletion } \\
(n=74)\end{array}$ & $\begin{array}{c}\text { Other Exon } 11 \\
\text { mutation }(n=89)\end{array}$ & $\begin{array}{l}\text { Other mutation } \\
\quad(n=16)\end{array}$ & $P$ value \\
\hline \multicolumn{6}{|l|}{ Gender } \\
\hline Male & $36(27.5)$ & $30(25.0)$ & $48(40.0)$ & $6(5.0)$ & \multirow[t]{2}{*}{0.293} \\
\hline Female & $36(27.5)$ & $44(33.6)$ & $41(31.3)$ & $10(7.6)$ & \\
\hline \multicolumn{6}{|l|}{ Age } \\
\hline$\leqq 65 \mathrm{yr}$ & $42(33.9)$ & $32(25.8)$ & $41(33.1)$ & $9(7.3)$ & \multirow[t]{2}{*}{0.249} \\
\hline$>65 \mathrm{yr}$ & $30(23.6)$ & $42(33.1)$ & $48(37.8)$ & $7(5.5)$ & \\
\hline \multicolumn{6}{|l|}{ Tumor site } \\
\hline Stomach & $48(28.4)$ & $49(29.0)$ & $61(36.1)$ & $11(6.5)$ & \multirow[t]{3}{*}{0.925} \\
\hline Small intestine & $22(28.9)$ & $22(28.9)$ & $27(35.5)$ & $5(6.6)$ & \\
\hline Others & $2(33.3)$ & $3(50.0)$ & $1(16.7)$ & $0(0.0)$ & \\
\hline \multicolumn{6}{|l|}{ Tumor size } \\
\hline$\leqq 10 \mathrm{~cm}$ & $55(27.2)$ & $62(30.7)$ & $70(34.7)$ & $15(7.4)$ & \multirow[t]{3}{*}{0.438} \\
\hline$>10 \mathrm{~cm}$ & $12(33.3)$ & $7(19.4)$ & $16(44.4)$ & $1(2.8)$ & \\
\hline unknown & $5(38.5)$ & $5(38.5)$ & $3(23.1)$ & $0(0.0)$ & \\
\hline \multicolumn{6}{|l|}{ Morphology } \\
\hline Spindle & $55(27.2)$ & $62(30.7)$ & $70(34.7)$ & $15(7.4)$ & \multirow[t]{4}{*}{0.438} \\
\hline Epithelioid & $12(33.3)$ & $7(19.4)$ & $16(44.4)$ & $1(2.8)$ & \\
\hline Mix & $5(38.5)$ & $5(38.5)$ & $3(23.1)$ & $0(0.0)$ & \\
\hline unknown & & & & & \\
\hline \multicolumn{6}{|l|}{ Mitosis } \\
\hline$\leqq 5 / 50 \mathrm{HPFs}$ & $29(26.4)$ & $29(26.4)$ & $44(40.0)$ & $8(7.3)$ & \multirow[t]{3}{*}{0.790} \\
\hline$>5 / 50 \mathrm{HPFs}$ & $31(32.3)$ & $29(30.2)$ & $31(32.3)$ & $5(5.2)$ & \\
\hline unknown & $12(26.7)$ & $16(35.6)$ & $14(31.1)$ & $3(6.7)$ & \\
\hline \multicolumn{6}{|l|}{ Risk group } \\
\hline Non-high risk & $45(25.0)$ & $52(28.9)$ & $69(38.3)$ & $14(7.8)$ & \multirow[t]{3}{*}{0.307} \\
\hline High risk & $22(37.9)$ & $17(29.3)$ & $17(29.3)$ & $2(3.4)$ & \\
\hline unknown & $5(38.5)$ & $5(38.5)$ & $3(23.1)$ & $0(0.0)$ & \\
\hline \multicolumn{6}{|l|}{ Adjuvant therapy } \\
\hline Yes & $13(14.6)$ & $30(33.7)$ & $38(42.7)$ & $8(9.0)$ & \multirow[t]{2}{*}{0.003} \\
\hline No & $59(36.4)$ & $44(27.2)$ & $51(31.5)$ & $8(4.9)$ & \\
\hline \multicolumn{6}{|l|}{ Recurrence status } \\
\hline Yes & $8(21.6)$ & $18(48.6)$ & $8(21.6)$ & $3(8.1)$ & \multirow[t]{2}{*}{0.033} \\
\hline No & $64(29.9)$ & $56(26.2)$ & $81(37.9)$ & $13(6.1)$ & \\
\hline \multicolumn{6}{|l|}{ Metastatic status } \\
\hline Yes & $7(11.7)$ & $30(50.0)$ & $19(31.7)$ & $4(6.7)$ & \multirow[t]{2}{*}{0.000} \\
\hline No & $65(34.0)$ & $44(23.0)$ & $70(36.6)$ & $12(6.3)$ & \\
\hline \multicolumn{6}{|l|}{ CD117 } \\
\hline Positive & $36(20.2)$ & $60(33.7)$ & $69(38.8)$ & $13(7.3)$ & \multirow[t]{3}{*}{0.000} \\
\hline Negative & $7(87.5)$ & $0(0.0)$ & $0(0.0)$ & $1(12.5)$ & \\
\hline unknown & $29(44.6)$ & $14(21.5)$ & $20(30.8)$ & $2(3.1)$ & \\
\hline
\end{tabular}

\section{Figures}


A

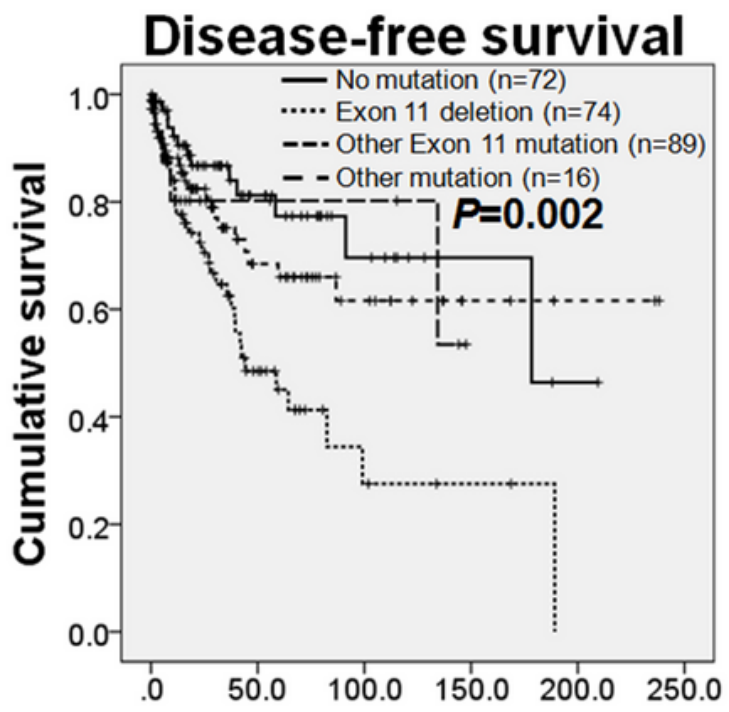

No. at risk

Month

No mutation

Exon 11 deletion

Other Exon 11 mutation

Other mutation

$\begin{array}{lccccc}72 & 25 & 9 & 3 & 1 & 0 \\ 74 & 18 & 4 & 2 & 0 & 0 \\ 89 & 28 & 13 & 4 & 2 & 0 \\ 16 & 5 & 4 & 0 & 0 & 0\end{array}$

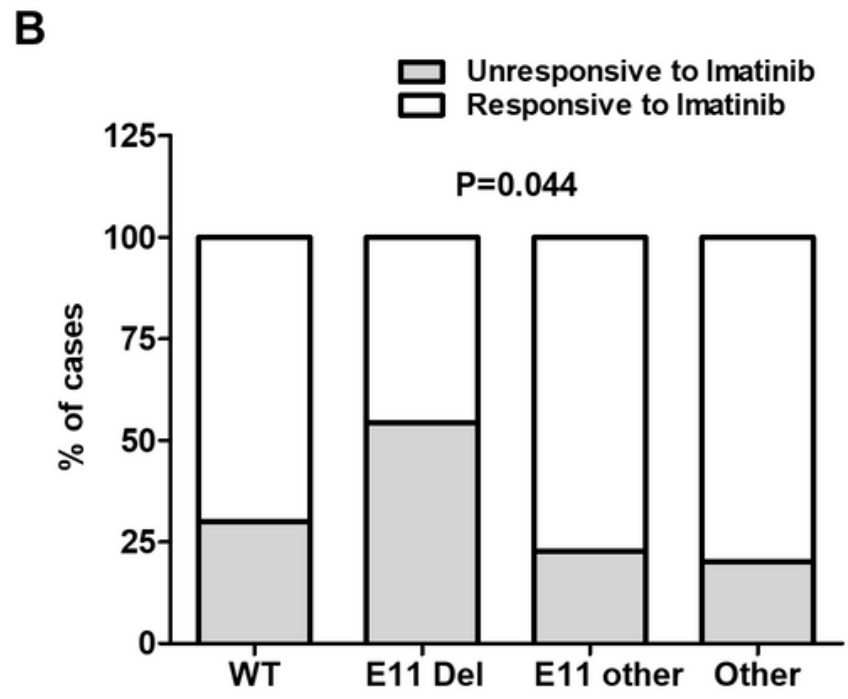

Figure 1

. C-KIT exon 11 deletions are associated with poor disease-free survival and IM resistance in GISTs. A, the correlation between c-KIT mutation types and disease-free survival was calculated using Kaplan-Meier survival analysis. B, the bar graph depicts the frequency distribution of IM resistance in GIST patients with different c-KIT mutation types. WT, E11, E11 other, and other indicate wild type c-KIT, c-KIT exon 11 deletion, other c-KIT exon 11 mutations, and other c-KIT mutations, respectively. 
A
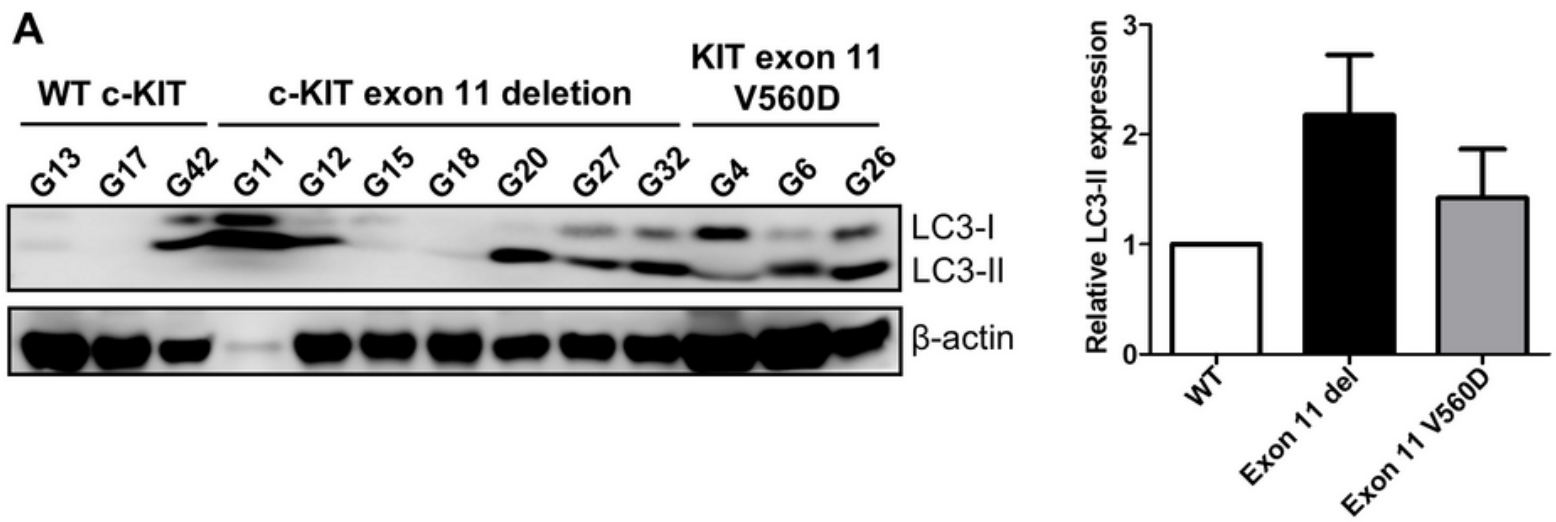

B

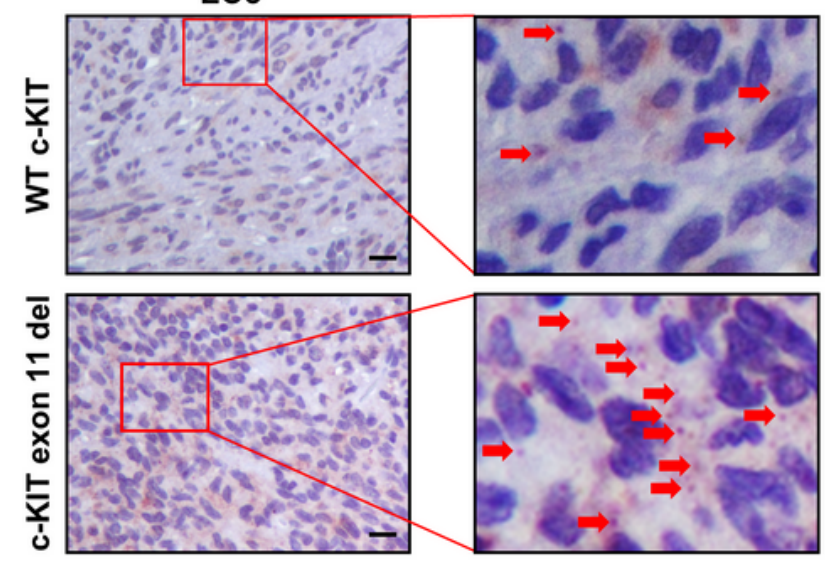

C

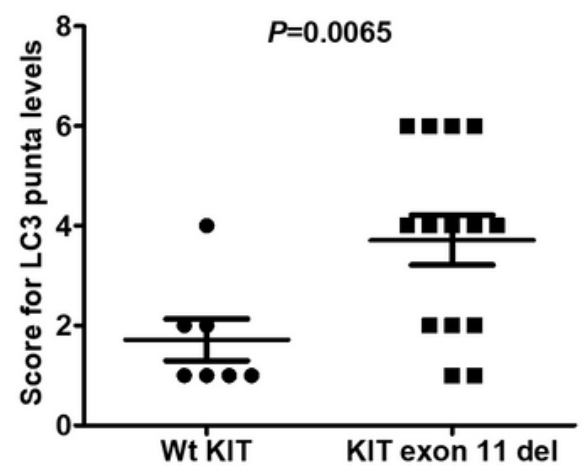

D

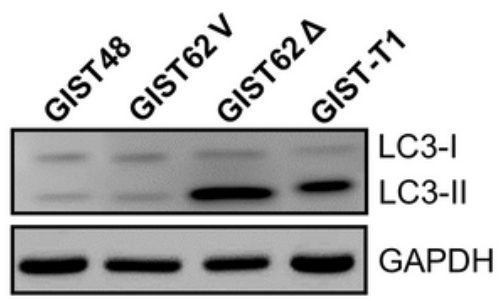

E

GIST62 V

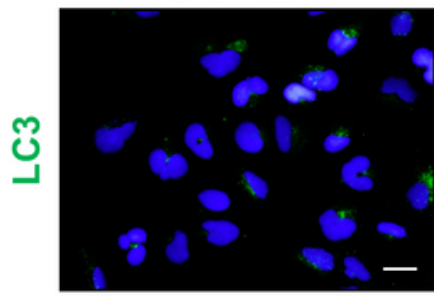

GIST62 $\Delta$

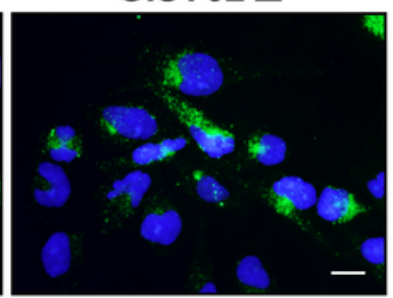

Figure 2

c-KIT exon 11 deletions induce autophagy in GIST cells. A, LC3 expression in tumor specimens from human GISTs harboring wild type c-KIT, c-KIT exon 11 deletion, and c-KIT exon 11 V560D mutation was analyzed by Western blotting. LC3-II expression was calculated as fold change relative to GISTs with wild type c-KIT, normalized to $\bigotimes$-actin. B, IHC staining for LC3 was performed on GISTs with wild type c-KIT and c-KIT exon 11 deletions. Red arrows indicate LC3 puncta. Magnification: 100区 (left panel). Scale bar: 20 
$\square \mathrm{m}$. Images on the right panel are high-magnification (400ロ) of areas outlined by red squares. C, the scores for LC3 puncta levels calculated according to the intensity and percentages of positive cells were compared between GISTs with wild type c-KIT and c-KIT exon 11 deletion. Each dot represents a sample and horizontal lines indicate means $\triangle \mathrm{SEM}$. $\mathrm{P}=0.0065$ versus wild type $\mathrm{c}-\mathrm{KIT}$, unpaired t-test. $\mathrm{D}, \mathrm{LC} 3$ expression in GIST48 cells, GIST62 V cells, GIST62 $₫$ cells, and G IST-T1 cells was determined by Western blotting. E, IF staining for LC3 was performed in GIST62 V cells and GIST62 $\otimes$ cells.

A

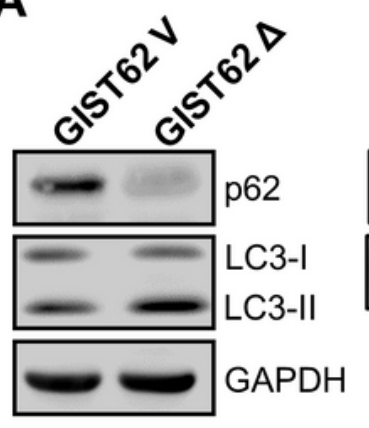

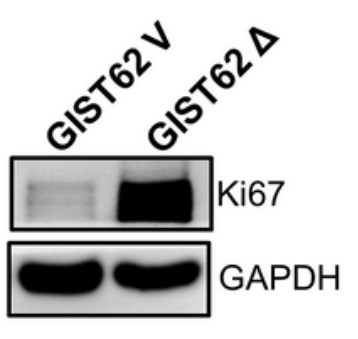

B

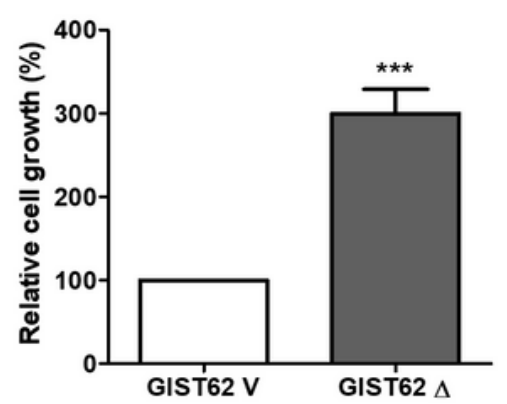

C

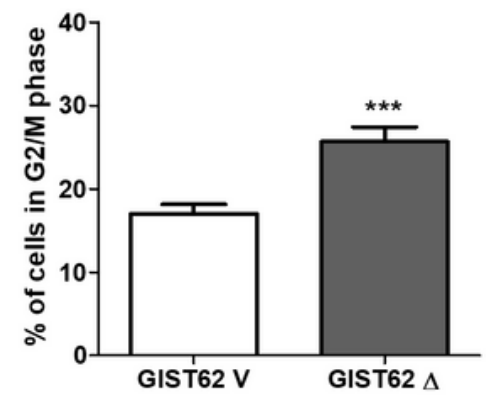

D

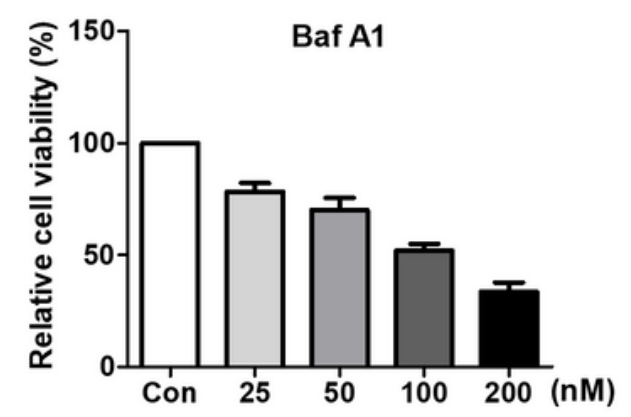

F

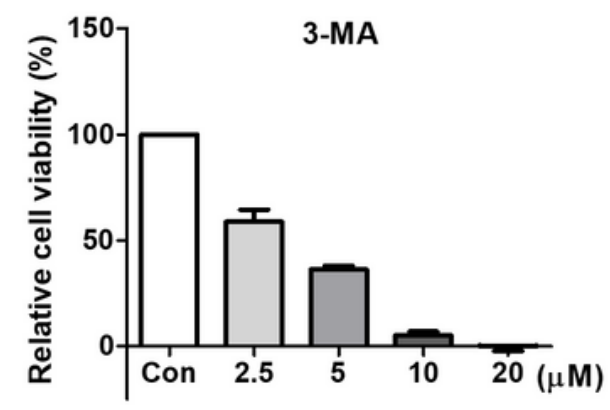

E

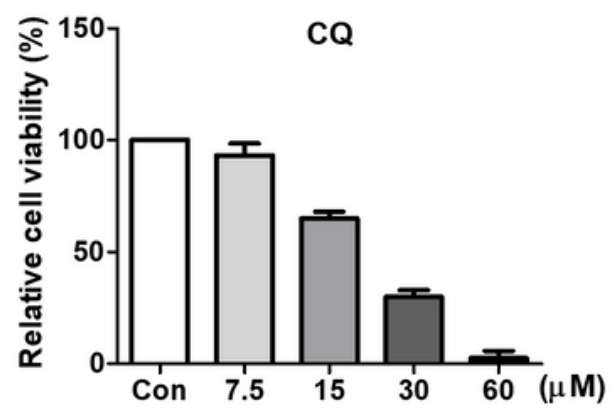

G

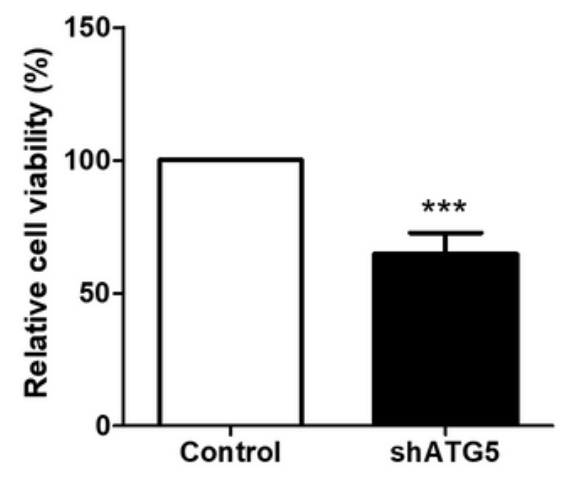

Figure 3 
Inhibition of autophagy decrease the viability of GIST cells with c-KIT exon 11 deletions. A, expression of p62, LC3, and Ki67 in GIST62 V cells and GIST62 $₫$ cells was measured by Western blotting. B, The viability of both GIST62 $\mathrm{V}$ and GIST62 $₫$ cells was analyzed by MTT assay. $* \star * ~ P<0.001$ versus GIST62 V cells, unpaired t-test. C, the percentage of cells of both GIST62 V and GIST62 $\otimes$ cells in the G2/M phase was analyzed by BrDU incorporation assay. ${ }^{* \star} P<0.001$ versus GIST62 V cells, unpaired t-test. $D, E$, and F, GIST62 $\otimes$ cells were treated with the autophagy inhibitors Baf A1, CQ, and 3-MA at indicated doses for 24 hours. The cell viability was measured by MTT assay. G, after knockdown of ATG5, the viability of GIST62 $\otimes$ cells was measured by MTT assay. ${ }^{* \star *} \mathrm{P}<0.001$ versus control cells, unpaired t-test. 
A

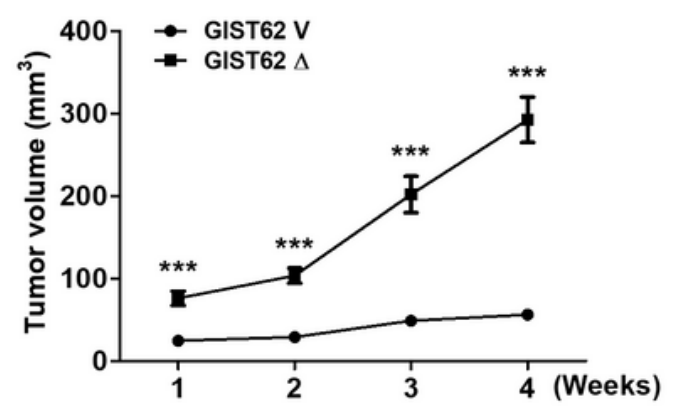

C

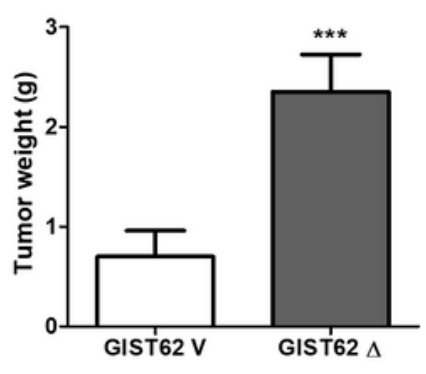

D

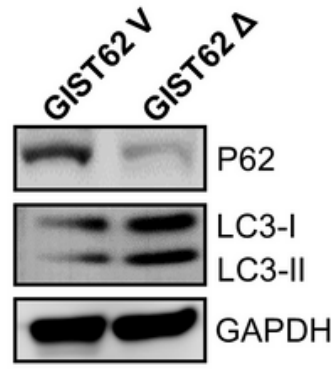

B

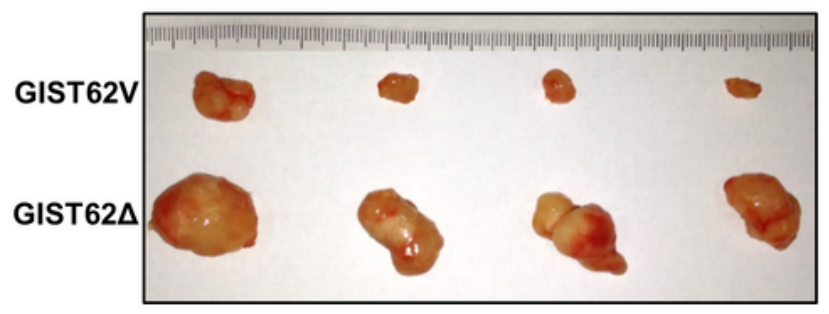

E

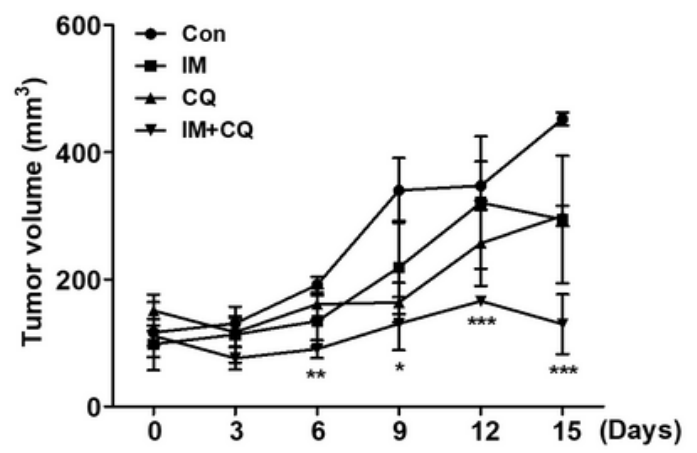

G
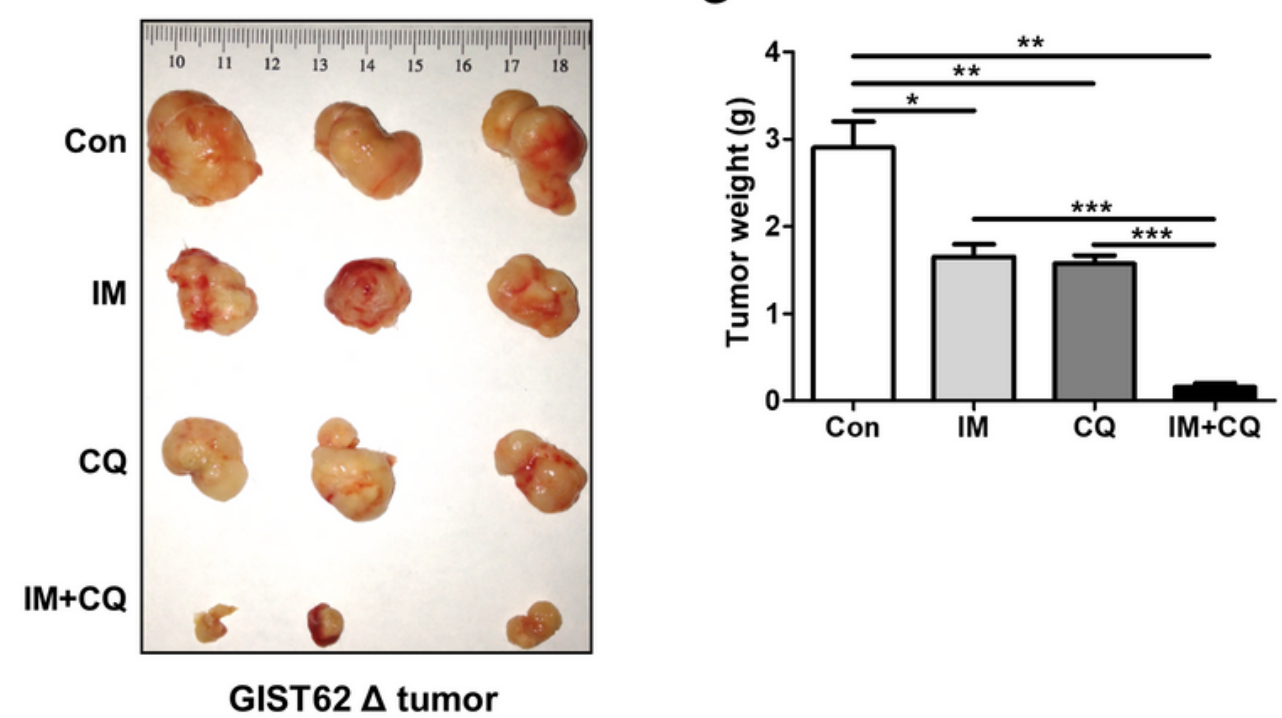

\section{Figure 4}

Autophagy inhibition reduces tumor growth of GIST cells harboring c-KIT exon 11 deletion and potentiates antitumor effect of IM. A, GIST62 V and GIST62 $\Delta$ cells were subcutaneously inoculated into SCID mice. Tumor volume was calculated weekly. The graph depicts the growth curves of tumors. ${ }^{\star} * \star \mathrm{P}<$ 0.001 versus GIST62 V cells, unpaired t-test. B, representative image of tumors dissected at the end of experiment from GIST62 V and GIST62 $\Delta$ cells is shown. C, tumor weight was measured at the end of the 
experiment. ${ }^{* \star *} \mathrm{P}<0.001$ versus GIST62 V cells, unpaired t-test. D, levels of autophagy in GIST62 V and GIST62 $\Delta$ tumors were confirmed by measuring LC3 II and p62 expression using western bloting. E, GIST62 $\Delta$ tumors were treated with imatinib $(40 \mathrm{mg} / \mathrm{kg})$, chloroquine $(\mathrm{CQ}, 60 \mathrm{mg} / \mathrm{kg})$, and their combination. The tumor volumes were measured once three days after treatments. F, representative image shows GIST62 $\Delta$ tumors receiving different treatments that were dissected at the end of experiment. G, bar graph depicts tumor weight of different treatment groups measured at the end of the

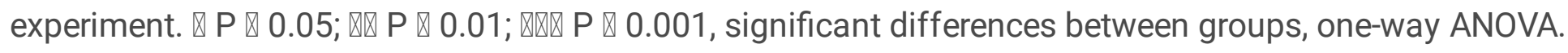

A

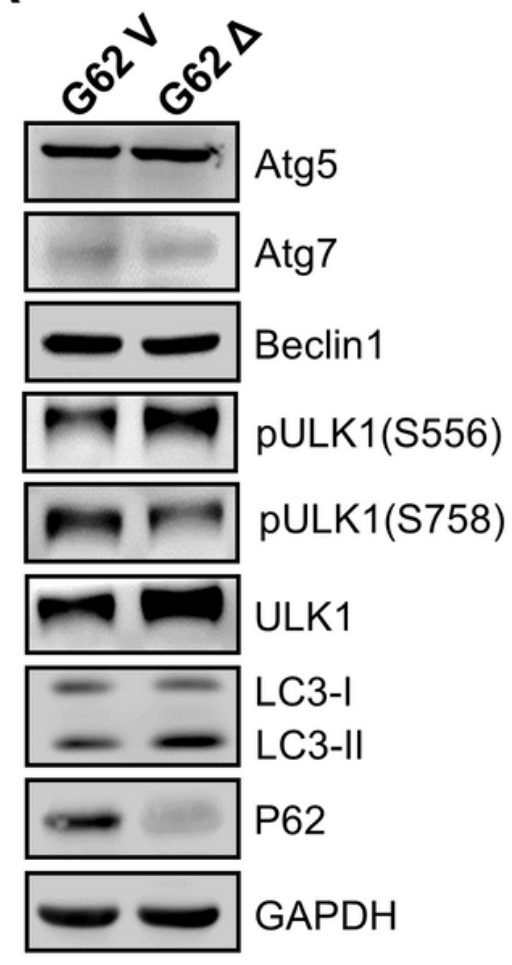

D

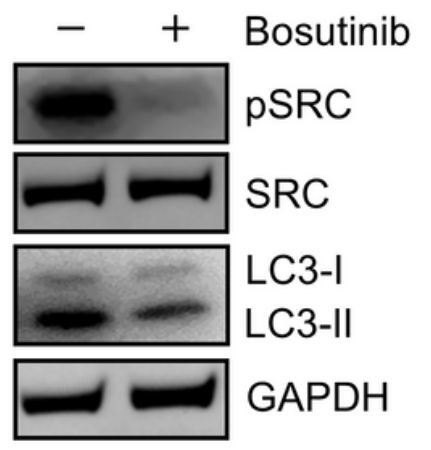

$\overline{\text { G62 } \Delta \text { cells }}$
B

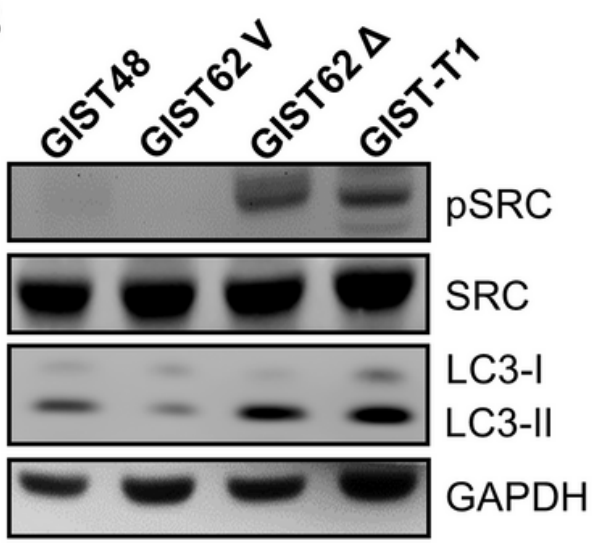

C

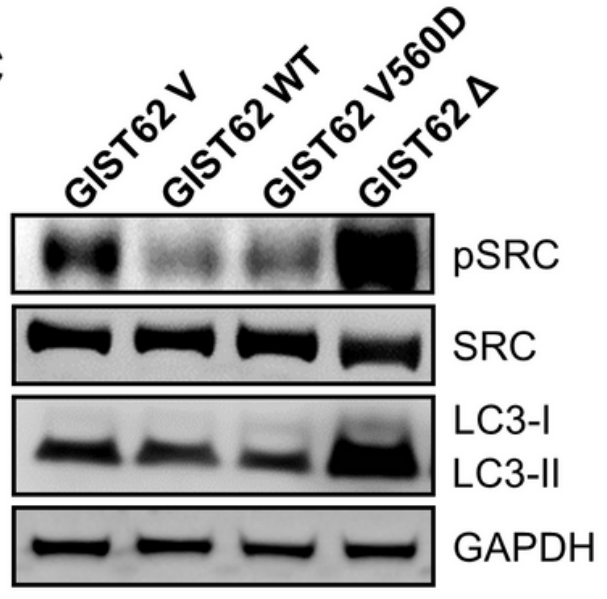

E

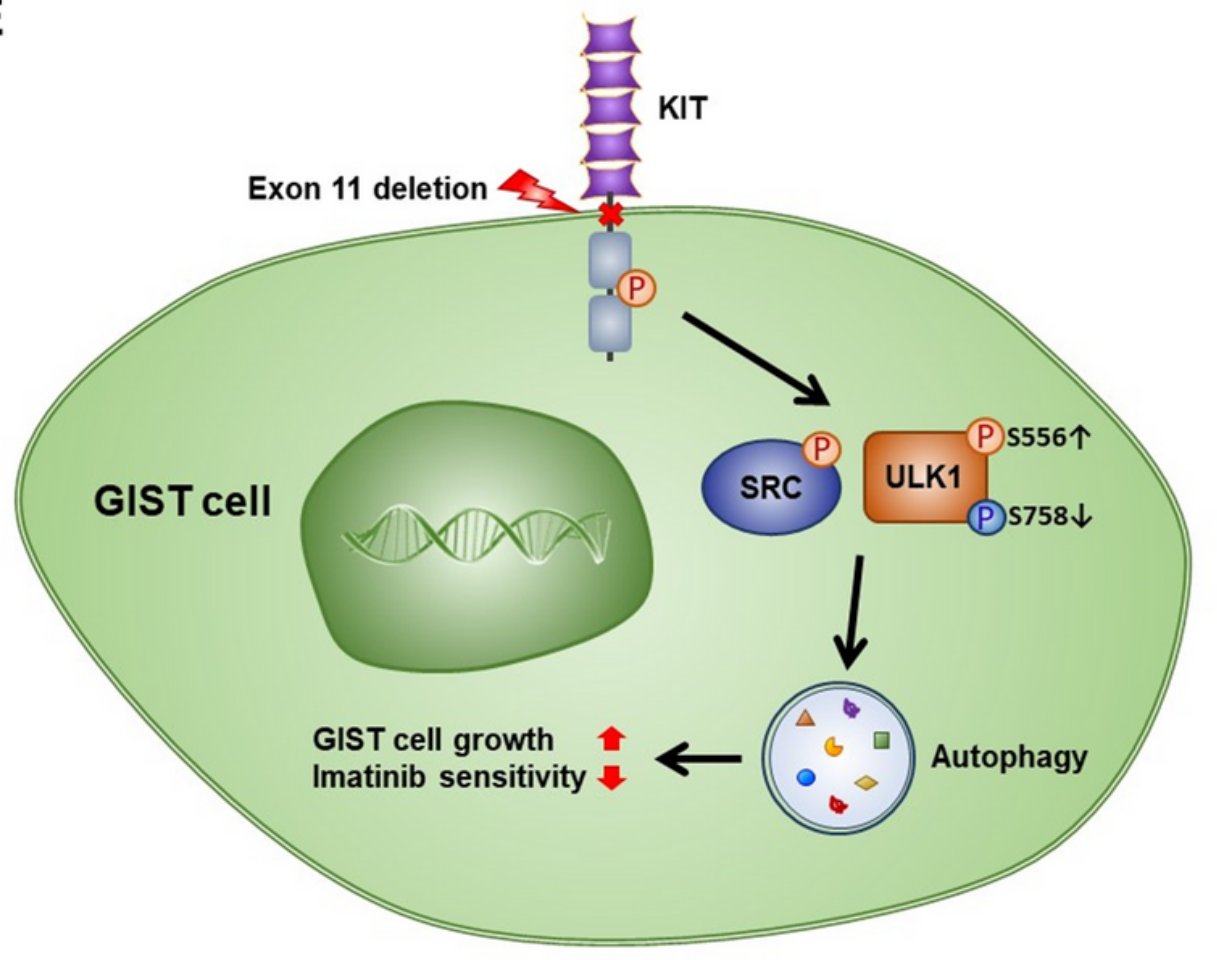

Figure 5 
ULK1 and SRC are involved in C-KIT exon 11 deletion-induced autophagy. A, whole cell lysates from GIST62 V and GIST62 $\Delta$ cells were subjected to Western blotting with the indicated antibodies. B, phsphorylation of SRC was measured by Western blotting in GIST 48 cells, GIST62 V cells, GIST62 $₫$ cells, and GIST-T1 cells. C, phsphorylation of SRC was determined by Western blotting in GIST 62-derived cell lines, GIST62 V cells, GIST62 WT cells, GIST62 V560D cells, and GIST62 $₫$ cells. D, GIST62 $₫$ cells were treated with the SRC inhibitor Bosutinib (10 $\square \mathrm{M})$ for 24 hours. Cell lysates were collected and subjected to Western blotting using the indicated antibodies. E, schematic representation of the mechanism by which c-KIT exon 11 deletion induces autophagy in GIST cells.

\section{Supplementary Files}

This is a list of supplementary files associated with this preprint. Click to download.

- SupplementalFigure1.jpg

- SupplementalFigure2.jpg 